\title{
Stimulated spin dynamics of polaritons in semiconductor microcavities
}

\author{
P. G. Lagoudakis, ${ }^{1}$ P. G. Savvidis, ${ }^{1}$ J. J. Baumberg, ${ }^{1}$ D. M. Whittaker, ${ }^{2}$ P. R. Eastham, ${ }^{2}$ M. S. Skolnick, ${ }^{3}$ and J. S. Roberts ${ }^{4}$ \\ ${ }^{1}$ Department of Physics \& Astronomy, University of Southampton, Southampton SO17 1BJ, United Kingdom \\ ${ }^{2}$ Cavendish Laboratory, University of Cambridge, Cambridge CB3 OHE, United Kingdom \\ ${ }^{3}$ Department of Physics, University of Sheffield, Sheffield S3 7RH, United Kingdom \\ ${ }^{4}$ Department of Electronic and Electrical Engineering, University of Sheffield, Sheffield S1 3JD, United Kingdom
}

(Received 25 February 2002; published 19 April 2002)

\begin{abstract}
Time-resolved polarization spectroscopy of polariton pair scattering in semiconductor microcavities enables complete measurement of the polariton spin dynamics. In addition to spin-preserving interactions previously reported, we observe two additional even stronger scattering processes, which mix polaritons of opposite spin. Because of the polaritons' bosonic character, this results in the stimulation of spin flips. Such mechanisms should allow realization of spin-sensitive interferometers.
\end{abstract}

DOI: 10.1103/PhysRevB.65.161310

PACS number(s): 71.36. $+\mathrm{c}, 42.65 .-\mathrm{k}, 78.45 .+\mathrm{h}, 78.67 .-\mathrm{n}$

The strong coupling of light to electronic transitions in semiconductors has recently become of great interest because of the additional interactions allowed. These interactions originate from the complete mixing of photons with hydrogenically bound electron-hole pairs, which produces mixed quasiparticles called exciton polaritons with radically different properties. ${ }^{1-3}$ In particular, pair scattering events between the polaritons become possible, which can be exploited for optical amplification, with gain per unit length greater than in any other material. ${ }^{4,5}$ These polaritonic scattering interactions are so strong because they are stimulated, in contrast to normal electronic scattering, which exhibits a fermionic saturation. This has allowed demonstration of a submicron optical parametric oscillator. ${ }^{6}$ Such interactions can be described either through nonlinear wave mixings ${ }^{7,8}$ or as the parametric scattering of pairs of electronic quasiparticles. ${ }^{9}$ While stimulated phonon-polariton scattering has been observed in bulk samples, ${ }^{10}$ only the exciton polaritons considered here are readily modified through sample design. Polaritons in these devices can condense into a ground state possessing macroscopic electronic coherence, resembling a superfluid or atomic condensate. ${ }^{6}$ Since the polaritons possess well-defined spin, magnetic-field-sensitive interferometers based on these coherent spin states should be feasible. ${ }^{3,11}$ However the crucial spin-dependent pair scattering needed for such spintronics devices is not well understood. ${ }^{12}$

In this paper we comprehensively analyze the polarization dynamics of the light emitted from microcavity samples, which are resonantly pumped at a critical angle to the normal, in order to understand the underlying spin dynamics of the polaritons. In this regime, strong angular asymmetries are observed in the photoluminescence ${ }^{13-15}$ produced by parametric scattering between polaritons with different in-plane momentum and energy. Strong nonlinearities in the emission are produced when this scattering is externally stimulated by seeding the final polariton state. This process has been shown to provide extremely strong gain $\sim 10^{6} \mathrm{~cm}^{-1}$, building up substantial polariton populations in the lowest-energy states, which emit normally from the sample. In previous studies, parametric scattering was found to be strongest when the spin of the interacting quasiparticles was identical. ${ }^{4}$ Recent cw measurements conversely show that elliptically polarized pumping produces stronger emission, however it remains unclear what processes can be involved. ${ }^{16}$ We show here that additional parametric interactions can occur with a flip of the spin. Because polaritons behave as bosons, this spin-flip process can be stimulated by occupation of the final spin state. Our results access a previously unobserved regime of parametric interactions in which polarization mode degeneracy introduces a new degree of freedom based on the relative phase of competing macroscopically coherent electronic states.

The semiconductor microcavity consists of two pairs of three $100-\AA \mathrm{In}_{0.06} \mathrm{Ga}_{0.94} \mathrm{As}$ quantum wells (QWs) in $100-\AA$ GaAs barriers, placed between 17 (20) pairs of $\mathrm{GaAs} / \mathrm{Al}_{0.18} \mathrm{Ga}_{0.82}$ As distributed Bragg reflectors on top (bottom). The optical cavity length is $\sim 3 \lambda_{e x} / 2$ and the cavity resonance $\left(\omega_{\text {cav }}\right)$ is tuned resonantly with the exciton energy $\omega_{e x}$. The sample is held in a cold-finger cryostat at a temperature of $10 \mathrm{~K}$. The strong coupling of exciton and cavity modes produces two additional polariton branches of which only the ${ }^{-}$-shaped lower polariton dispersion $\omega_{L P}$ is of concern here [Fig. 1(a)]. Each branch is doubly spin degenerate $(J= \pm 1)$, with circularly polarized light of each helicity able to couple to one spin only. Thus we can simply track polariton spins using polarization analysis of the emitted photons.

In order to track the spin dynamics of the parametric scattering mechanism we perform our measurements in a regime that shows strong stimulated gain. ${ }^{4}$ Pulsed experiments allow us to disentangle the different scattering processes, while remaining below threshold for parametric oscillation. A pump pulse, incident at the critical angle of $16.5^{\circ}$ injects polaritons at the point of inflection on the dispersion relation, and a normally incident probe pulse seeds polaritons with zero inplane momentum [Fig. 1(a)]. This seed is amplified by over two orders of magnitude, producing emerging beams at $0^{\circ}$ ("signal") and $35^{\circ}$ ("idler"). The incident pulses are derived from a 100-fs mode-locked Ti:sapphire laser and are separately spectrally filtered inside zero-dispersion grating compressors in order to selectively excite the lower polariton branch. The polarization of each 3-ps pulse is set by tunable wave plates and arranged to compensate Fresnel losses at the 
(a)
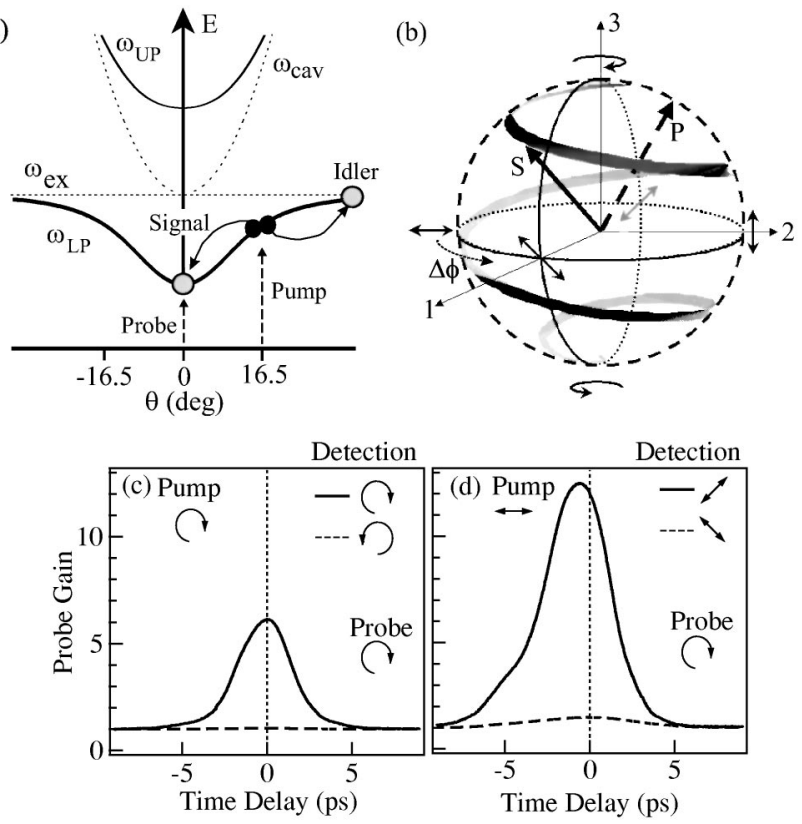

FIG. 1. (a) Dispersion relations of exciton, cavity photon, upper and lower polaritons, showing the dominant pair scattering for pump polaritons. (b) Poincaré sphere for representing polarizations: right/left circular at $P_{3}= \pm 1$ and linear around the equator, $P_{3}=0$. In experiments, the pump follows the dashed meridian, while the signal emission follows the solid spiral. (c,d) Emitted signal for (c) cocircular pump and probe, and (d) linear pump and circular probe, as a function of their time delay, polarization analyzed as specified.

sample surface. Throughout the measurements we keep the

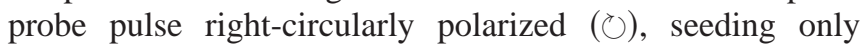
spin-up polaritons $(\Uparrow)$. The spectral, temporal, and polarization composition of the emitted light is analyzed as the pump polarization is systematically varied from right to left circular passing through intermediate states of elliptical, horizontal, and vertical polarization. This varies the ratio of spin-up to spin-down polaritons injected by the pump, while keeping the total polariton density fixed. To keep track of the spin states we use the Stokes parameters defining the vector $\mathbf{P}$ on the Poincaré sphere

$$
P_{1}=\frac{I_{\uparrow}-I_{\hookleftarrow}}{I_{\uparrow}+I_{\hookleftarrow}}, \quad P_{2}=\frac{I_{\nearrow}-I_{\searrow}}{I_{\nearrow}+I_{\searrow}}, \quad P_{3}=\frac{I_{\circlearrowleft}-I_{\circlearrowright}}{I_{\circlearrowleft}+I_{\circlearrowright}},
$$

where $I_{\uparrow, \leftrightarrow, \nearrow, \backslash}$ are the intensities of linear components at $0^{\circ}, 90^{\circ}, \pm 45^{\circ}$ to the horizontal, and $I_{\circlearrowright, \circlearrowleft}$ are the circular components. On the Poincare sphere the Stokes vector of the injected pump $\mathbf{P}$ moves around a meridian passing through horizontal and vertical polarizations [Fig. 1(b), dashed]. In order to keep track of how the polarization of the emitted light evolves as $\mathbf{P}$ is varied, we perform three independent measurements that correspond to the three orthogonal axes of the Poincare sphere. By judicious settings of $\lambda / 2, \lambda / 4$ plates and a polarizing beam splitter we thus extract the signal (S) and idler (I) Stokes vectors as a function of the ratio of injected spin-up to spin-down polaritons, which is set by the pump ellipticity component $P_{3}$.
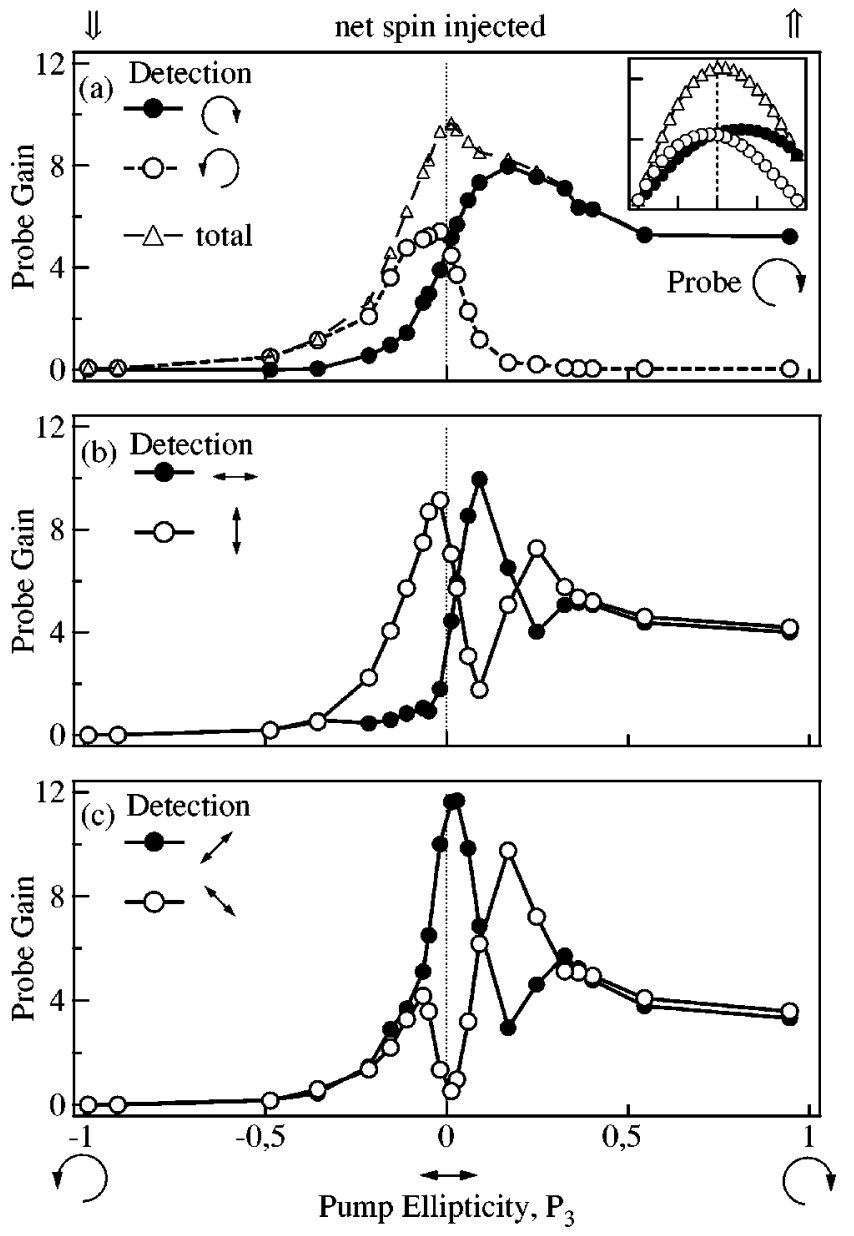

FIG. 2. Emitted signal intensities decomposed into (a) circular, (b) linear, and (c) linear diagonal, polarizations, as a function of the ellipticity of the pump. The probe is right-circularly polarized. The inset in (a) shows predictions from the model.

If circularly polarized pulses are used, injecting polaritons of one specific spin, stimulated gain of the probe polaritons is seen only for cocircularly polarized pump and probe near zero time delay [Fig. 1(c)]. In this case the emitted signal is completely circularly polarized indicating the absence of significant polariton spin flips within their 3 ps radiative lifetime. However, when the pump pulse is horizontally polarized, which injects equal populations of spin-up and spindown polaritons, the emitted signal is linearly polarized at $45^{\circ}$ [Fig. 1(d)]. Moreover, the gain is nearly twice as large for this configuration even though the density of polaritons with the same spin as the probe is halved. Despite the large variation in gain magnitude in these two cases, the speed of response is very similar and the gain is maximized at the same (within $1 \mathrm{ps)}$ ) pump-probe time delay [Figs. 1(c,d)].

To explore this phenomenon in more detail, Fig. 2 plots the intensity of each polarization component as a function of the pump ellipticity $P_{3}$, which corresponds to the ratio of spin-up to spin-down polaritons injected (termed the net spin injected). The total gain is maximized for the linear pump $\left(P_{3}=0\right)$, while to maximize emission from the spin-up seeded polaritons requires an elliptical pump, $P_{3}=0.15$ [Fig. 2(a)]. Light also emerges from nonseeded spin-down polari- 


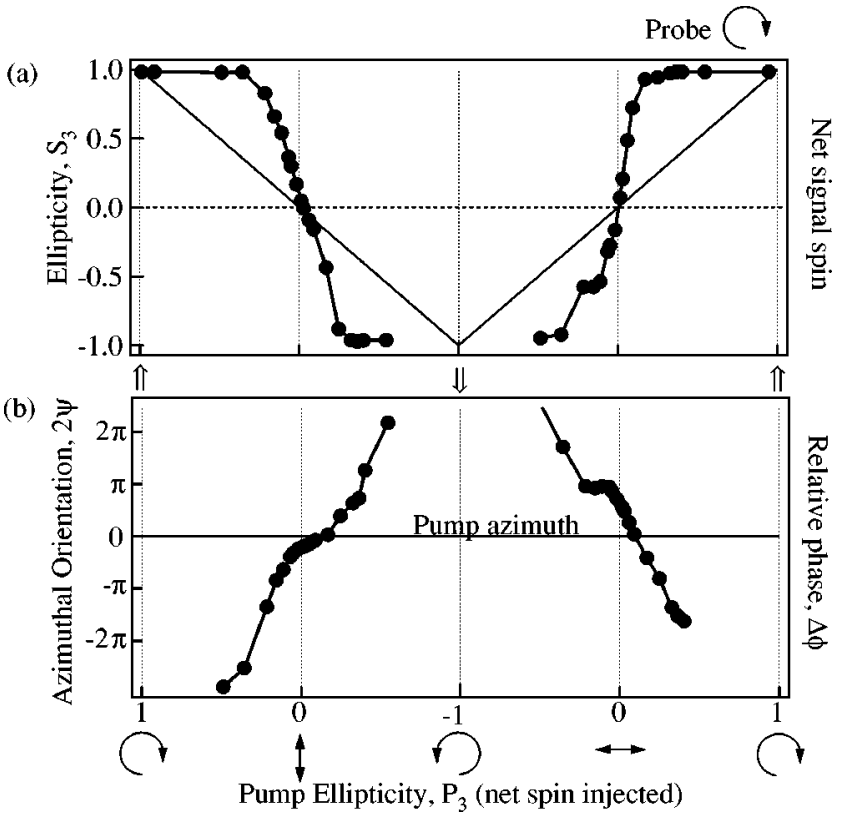

FIG. 3. Signal emission as a function of pump ellipticity, decomposed into (a) ellipticity and (b) azimuthal orientation of polarization ellipse.

tons: the intensity of this unexpected contribution can be even larger than that from the spin-up polaritons, and their emissions are matched when the pump injects equal spin populations. Examining the linear components [Figs. 2(b,c)] shows that they rapidly oscillate in strength near $P_{3} \sim 0$, changing from near vertical to near horizontal with only a 5\% change in net spin population. As seen in Fig. 1(d), the emission is completely diagonally polarized at $P_{3}=0$. This graphically demonstrates the necessity of complete polarization analysis to resolve the true spin dynamics of the dynamical processes. The simple description of spin-preserving parametric scattering completely fails to predict this large enhancement.

To summarize the polarization behavior, we plot the net spin of the signal polaritons, evidenced by the ellipticity of their emission, $S_{3}$ [Fig. 3(a)]. The idler emission is 50 times weaker than the signal emission due to exciton scattering processes competing for these higher-energy polaritons, ${ }^{17}$ but follows a similar trend. The steep gradient of Fig. 3(a) demonstrates the delicate spin balance controlled by the pump - a small net spin in the pump polaritons creates a much larger net spin in the signal polaritons.

Due to the stimulation process, the phase of each parametrically scattering polariton is set by the phase of those polaritons already in the final state. Thus the two separate spin populations of signal polaritons can have different macroscopic phases, $\phi_{\Uparrow, \Downarrow}$. As well as the ratio of spin populations in the signal, we track their relative phase, $\Delta \phi=\phi_{\Uparrow}$ $-\phi_{\Downarrow}$, which corresponds to the azimuthal orientation of the emitted polarization ellipse, $\psi=\tan ^{-1}\left(S_{2} / S_{1}\right)=\Delta \phi / 2$ [Fig. 3(b)]. The axis of the signal polarization ellipse twists rapidly depending on the net spin injected into the system, $\Delta \phi \propto S_{3}$. On the Poincaré sphere, $2 \psi$ is the longitude of the Stokes vector, which rotates through more than two full

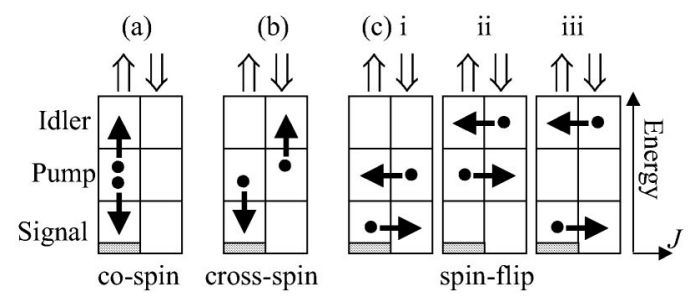

FIG. 4. Parametric scattering between pump, signal, and idler: (a) spin preserving, (b) cross spin, and (c) spin flip. The probe seeds the spin-up signal.

revolutions as the majority spin passes from up $(\Uparrow)$ to down $(\Downarrow)$. This dramatic contrast with the fixed pump azimuthal orientation is seen in the spiraling trajectory of the signal Stokes vector [Fig. 1(c)]. We have confirmed that the complete set of these observations are independent of the pump power, strongly suggesting a fundamental origin in the microscopic interactions.

Our data implies that fresh processes occur when polaritons of both spins are simultaneously present in the sample. Because of the shape of the polariton dispersion relation, only two-particle scattering processes can satisfy energy and momentum, allowing us to restrict the interactions to third order. $^{17,9}$ The nonlinearity is driven by nonlocal dipoledipole Coulomb interactions between colliding polaritons (which behave as interacting bosons at these densities). ${ }^{18}$ Focusing discussion only on the dominant polariton spin populations at signal, idler, and pump wave vectors allows decomposition of the basic parametric processes using six states (Fig. 4). While the spin-preserving process (a) has been seen, ${ }^{4}$ two previously unobserved processes are identified: a cross-spin scattering (b) in which pump polaritons of opposite spin collide and a stimulated spin flip (c) in which elastic collisions between polaritons mutually flip their spins at a rate enhanced by the occupation of the final spin states. Surprisingly, the cross-spin process (b) must be more than twice as strong as (a), in order to match the experimental observation of strongest gain for the linearly polarized pump. Otherwise it would always be favorable to concentrate the pump polaritons in the same spin state as the seeded polaritons. By similar reasoning, observation of strong emission from spin-down signal polaritons requires strong stimulated spin flips, (c). The direct observation of these spin anomalies proves the dramatic influence of extra spin-cooperative parametric processes.

Incorporating the parametric terms (a)-(c) from Fig. 4 into the previously derived model ${ }^{9}$ results in six equations for the complex signal, idler, and pump fields, $s, i, p$, driven by the injected fields $f$,

$$
\begin{gathered}
j \dot{s}_{\Uparrow}=\delta_{s} s_{\Uparrow}+a i_{\Uparrow}^{\dagger} p_{\Uparrow}^{2}+b i_{\Downarrow}^{\dagger} p_{\Uparrow} p_{\Downarrow}+c s_{\Downarrow}\left(p_{\Downarrow}^{\dagger} p_{\Uparrow}+i_{\Downarrow}^{\dagger} i_{\Uparrow}\right)+f_{\Uparrow}^{\text {seed }}, \\
j \dot{i}_{\Uparrow}=\delta_{i} i_{\Uparrow}+a s_{\Uparrow}^{\dagger} p_{\Uparrow}^{2}+b s_{\Downarrow}^{\dagger} p_{\Uparrow} p_{\Downarrow}+c i_{\Downarrow}\left(p_{\Downarrow}^{\dagger} p_{\Uparrow}+s_{\Downarrow}^{\dagger} s_{\Uparrow}\right), \\
j \dot{p}_{\Uparrow}=\delta_{p} p_{\Uparrow}+2 a p_{\Uparrow}^{\dagger} s_{\Uparrow} i_{\Uparrow}+b\left(s_{\Uparrow} i_{\Downarrow}+s_{\Downarrow} i_{\Uparrow}\right) p_{\Downarrow}^{\dagger} \\
+c\left(s_{\Uparrow} s_{\Downarrow}^{\dagger}+i_{\Uparrow} i_{\Downarrow}^{\dagger}\right) p_{\Downarrow}+f_{\Uparrow}^{\text {pump }}
\end{gathered}
$$


along with the equivalents with reversed spin states. Here $\delta_{s, i, p}=\Delta \omega_{s, i, p}+j \gamma_{s, i, p}$ contain the intensity-dependent detunings of the emission frequencies from the lower polariton branch, $\Delta \omega$, and the polariton decay rates $\gamma$ (and $j=\sqrt{-1}$ ). Numerical solutions of this model successfully predict that the largest gain results from linear pump polarizations, provided $b \sim c \sim 2 a$. This factor of 2 arises because for two colliding spin-antiparallel polaritons, both a triplet $(J=2)$ and singlet $(J=0)$ pathway are available. ${ }^{19}$ The stimulated spinflip scattering is thus indeed stronger than the spinpreserving process.

Previous demonstrations of peculiar microcavity spin properties using $\mathrm{cw}$ excitation have suggested that biexcitonic resonances are responsible. ${ }^{16}$ Although biexcitons are seen in pulsed measurements on QWs, ${ }^{7,20}$ they are absent in the low-density stimulated regime. Bound biexcitons are unlikely to dominate because the depth of the polariton trap at $k=0$ exceeds the biexciton binding energy and biexcitons involve exciton states out to very large $k$ (due to the small Bohr radius), while the parametric interactions remain localized at very small $k$. Indeed, our results are not strongly influenced by changing the detuning of the microcavity, suggesting the lack of a resonant biexciton interaction. However, simple calculations of the spin-antiparallel exciton-exciton interaction (which lead to coefficients $b, c$ ) show that it should be negligible ${ }^{19}$ - this issue remains to be resolved.

This spin-sensitive parametric scattering reveals a fine balance between the amplitude and phase of the spinantiparallel signal polariton populations. Although many aspects of our observations are reproduced [Fig. 2(a)], the model still fails to account for the phase difference between the two signal populations, $\Delta \phi$. In particular although it predicts a rapid change in azimuthal orientation around $P_{3}=0$, it cannot produce a $45^{\circ}$ linearly polarized signal from a hori- zontal linear pump. However it does show that the phase shift between spin-up and spin-down signal polaritons can differ from the relative phase shift imposed on the pump polaritons, $S_{1} \neq P_{1}=0$. In addition, experimentally the enhancement due to the spin-flip parametric scattering is found only close to the linear pump condition, whereas the model predicts that this can exist over a wider range of pump circularities. We suggest that additional spin-dependent energy shifts of the polariton states are responsible for this discrepancy. ${ }^{19}$

The emergence of two populations of polaritons with a specific electronic phase difference $\Delta \phi$ is reminiscent of Josephson coupling between two superconductors. Instead of condensates of Cooper pairs, cw-pumped semiconductor microcavities (at this critical angle) produce nonequilibrium condensates composed of signal polaritons. ${ }^{6}$ The macroscopic phase of each condensate is set by a spontaneous symmetry breaking and is not locked to the pump optical phase. A Josephson junction allows weak tunneling between two condensates with a magnitude set by their phase difference. With the possibility of two different spin populations, which are linked by spin-flip parametric scattering, the microcavity system mirrors that of a Josephson junction. The possibility of an optical Josephson device is of general interest for its sensitive interferometric applications, ${ }^{3,11}$ and we are actively investigating one of these. In addition, spin coherences and stimulated spin flips are of interest for developments in spintronics.

We are very grateful for critical comments from D. Martin, A. Kavokin, G. Malpuech, and C. Cuiti, and acknowledge the support of the HEFCE JR98SOBA, EC CLERMONT HPRN-CT-1999-00132, and EPSRC GR/ N18598.
${ }^{1}$ G. Khitrova, H.M. Gibbs, F. Jahnke, M. Kira, and S.W. Koch, Rev. Mod. Phys. 71, 1591 (1999).

${ }^{2}$ M.S. Skolnick, T.A. Fisher, and D.M. Whittaker, Semicond. Sci. Technol. 13, 645 (1998).

${ }^{3}$ J.J. Baumberg, in Semiconductor Spintronics and Quantum Computation, edited by D.D. Aschwalom and N. Samarth (SpringerVerlag, Berlin, 2001), Chap. 2.

${ }^{4}$ P.G. Savvidis et al., Phys. Rev. Lett. 84, 1547 (2000).

${ }^{5}$ G. Dasbach et al., Phys. Rev. B 62, 13076 (2000).

${ }^{6}$ J.J. Baumberg et al., Phys. Rev. B 62, R16 247 (2000).

${ }^{7}$ M. Kuwata-Gonokami et al., Phys. Rev. Lett. 79, 1341 (1997); M. Shirane et al., Phys. Rev. B 58, 7978 (1998).

${ }^{8}$ J.J. Baumberg, A. Armitage, M.S. Skolnick, and J.S. Roberts, Phys. Rev. Lett. 81, 661 (1998).

${ }^{9}$ C. Ciuti, P. Schwendimann, B. Deveaud, and A. Quattropani, Phys. Rev. B 62, R4825 (2000).
${ }^{10}$ Y.R. Shen, Principles of Nonlinear Optics (Wiley, New York, 1984), Chap. 10.

${ }^{11}$ M. Aihara and T. Iida, Phys. Rev. Lett. 77, 3597 (1996).

${ }^{12}$ M.D. Martin, L. Vina, J.K. Son, and E.E. Mendez, Solid State Commun. 117, 267 (2001).

${ }^{13}$ P.G. Savvidis et al., Phys. Rev. B 62, R13 278 (2000).

${ }^{14}$ J. Erland et al., Phys. Rev. Lett. 86, 5791 (2001).

${ }^{15}$ M. Müller, J. Bleuse, and R. Andre, Phys. Rev. B 62, 16886 (2000).

${ }^{16}$ A.I. Tartakovskii et al., Phys. Rev. B 62, R13 298 (2000).

${ }^{17}$ P.G. Savvidis et al., Phys. Rev. B 64, 075311 (2001).

${ }^{18}$ C. Ciuti, V. Savona, C. Piermarocchi, A. Quattropani, and P. Schwendimann, Phys. Rev. B 58, 7926 (1998).

${ }^{19}$ A. Kavokin and G. Malpuech (private communication).

${ }^{20}$ M. Saba et al., Phys. Rev. Lett. 85, 385 (2000). 